\title{
Selection of the elastic scattering events in interactions of the NICA colliding proton (deuteron) beams
}

\author{
Vasily Sharov ${ }^{1, \star}$ \\ ${ }^{1}$ Veksler and Baldin Laboratory of High Energy Physics, Joint Institute for Nuclear Research, Dubna, Russia
}

\begin{abstract}
The features of the kinematics of elastic $p p(d d)$ scattering in the collider system, as well as some issues concerning registration and selection of elastic scattering events in the NICA colliding beams are considered. Equality and the opposite direction of the scattered particle momenta provide a powerful selection criterion for elastic collisions. Variants of the organization of the trigger signal for recording tracks of secondary particles and $D A Q$ system are given. The estimates of the characteristics of elastic $N N$ processes are obtained from available $d \sigma / d \Omega_{C M}$ data for the elastic $p p$ and $n p$ scattering. The paper presents examples of simulations using the Monte-Carlo of elastic $p p$ scattering in the colliding proton beams and quasi-elastic $n p$ scattering in the colliding deuteron beams and evaluates the outputs of these processes at the NICA collider.
\end{abstract}

\section{Introduction}

The NICA collider [1] will provide the colliding beams of polarized protons and deuterons with the energy range of $T_{p}=5 \div 12.6 \mathrm{GeV}\left(\sqrt{s_{p p}}=12 \div 27 \mathrm{GeV}\right)$ for protons and $T_{d}=2 \div 5.5 \mathrm{GeV}\left(\sqrt{s_{N N}}=\right.$ $4 \div 13 \mathrm{GeV}$ ) for deuterons. Obtaining new data on spin-dependent observables in elastic $N N$ and $d d$ scattering at the colliding beams energies $T_{p, d}$ above a few $G e V$ could be the priority area of $V B L H E P$ research at the NICA collider.

In our previous reports and publications [2-4] we have considered opportunities of studying the spin $1 / 2$ and 1 observables in the elastic interactions of the colliding proton and deuteron beams of the NICA collider. It has been shown that in elastic interactions of colliding proton beams one can measure energy and angular dependencies of the analyzing powers of the reactions with one polarized beam $A_{\text {oono }}$ or $A_{\text {ooon }}$, and the spin correlation parameters $A_{\text {ook }}$ and $A_{\text {oonn }}$ in collisions of both polarized proton beams. In elastic interactions of the colliding deuteron beams it is possible to obtain a complete set of energy and angular dependencies of components of the vector and tensor analyzing powers $A_{y}$, $A_{y y}$, and $A_{x x}$ for the $d N$ and $d d$ reactions. The planned luminosity of the polarized colliding $p p$ and $d d$ beams will allow one to reach a sufficiently high counting rate of the elastic events in these measurements. The use of the colliding polarized $p p$ and $d d$ beams for the spin-dependent observables research will have a number of significant advantages in comparison with the experiments on the "fixed" target. The angular acceptance of the collider detector covers the full solid angle $4 \pi$ radians when the wide ranges of the energies of the $p p, d d$ interactions and the 4-momentum transfer squared are available.

\footnotetext{
^e-mail: vsharov41@mail.ru
} 
To obtain the values of the spin-dependent $p p$ or $d d$ observables, it is necessary to measure the asymmetry of the outputs of the processes under study, i.e. - the ratio of the difference to the sum of the elastic differential cross sections with opposite signs of polarization of the colliding particles (or right-left asymmetry of the outputs for the same sign of the polarization of the beams).

In this article we discuss the kinematics of the elastic $p p$ and $d d$ scattering processes in the collider system and some of the issues concerning the registration and selection of elastic events. The kinematics features are discussed in the 2-nd section of the paper. The 3-rd section presents the Spin Physics Detector $(S P D)$ elements and Monte Carlo $(M C)$ simulations of scattering events. The fourth section gives the examples of the $M C$ simulations for elastic events in the collider system using the existing experimental data on $d \sigma / d \Omega$ of elastic $p p$ and $n p$ collisions.

\section{The kinematics of elastic $p p$ and $d d$ scattering in the collider system}

To determine possible criteria for the selection of elastic events in interactions of colliding beams, we consider the features of the two-particle $1+2 \rightarrow 3+4$ kinematics in the collider system. The coordinate system of the collider is simultaneously the laboratory system and Centre-of-Mass system of the colliding particles. The total momentum of the colliding particles and the total momentum of the reaction products are equal to zero. In this case, the momenta of incoming and outgoing particles are equal to each other $\left|\mathbf{p}_{1}\right|=\left|\mathbf{p}_{2}\right|=\left|\mathbf{p}_{3}\right|=\left|\mathbf{p}_{4}\right|=\left|\mathbf{p}_{\text {beam }}\right|$ and are oriented in the opposite directions $\mathbf{p}_{1} \leftrightarrows \mathbf{p}_{2}$ and $\mathbf{p}_{3} \leftrightarrows \mathbf{p}_{4}$. The equality and opposite direction of the outgoing particle momenta provide a powerful criterion to select elastic $p p$ and $d d$ events in the coordinate system of the collider. A

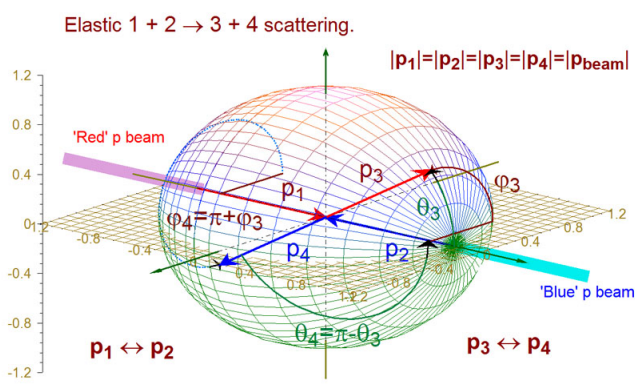

(a)

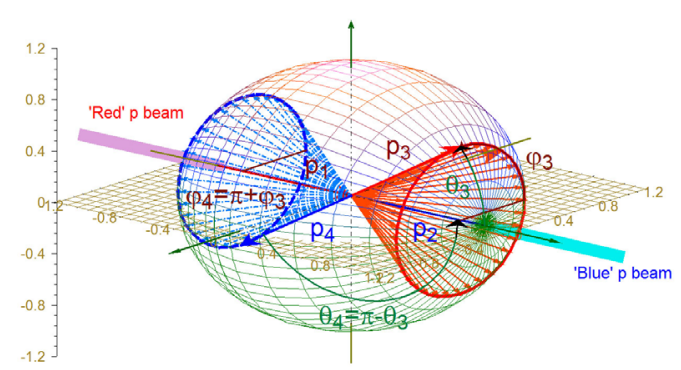

(b)

Figure 1. (a) Three-dimensional region of possible values and orientations of the particle momenta in the elastic $p p$ and $d d$ collisions at the NICA collider. (b) The distributions of the outgoing particles momenta are isotropic in the azimuthal direction.

three-dimensional range of possible values and orientation of the momentum of the particles in elastic $p p$ and $d d$ collisions in the collider represent a sphere with the radius value of $R=\left|\mathbf{p}_{\text {beam }}\right|$. Figure 1a. The direction of the momenta of secondary particles is determined by the values of polar $\theta_{3}$ and $\theta_{4}$ and azimuthal $\varphi_{3}$ and $\varphi_{4}$ angles of scattering. $>$ From the condition $\mathbf{p}_{3} \rightleftarrows \mathbf{p}_{4}$ it follows that the relation between the polar and azimuthal angles of the outgoing particles should be following: $\theta_{4}=\pi-\theta_{3}$ and $\varphi_{4}=\pi-\varphi_{3}$. The probability of the certain orientation of the $\mathbf{p}_{3}$ and $\mathbf{p}_{4}$ vectors is determined by the value of 4-momentum transfer squared $t_{1,3}$ from particle 1 to 3 or from particle 2 to 4 . The value of $t_{1,3}$ for $p p$ collisions in the $C M$ system is defined by the following expression: $t_{1.3}=2 m_{p}^{2}-2 E_{p}^{2}+2 p_{p}^{2} \cos \left(\theta_{1,3}\right)$. It is obvious that for the fixed value of $t_{1.3}$, i.e. at the fixed angle $\theta$, the output of the elastic processes will be the same at any value of the azimuthal angle. In the collisions of unpolarized particles the spatial distribution of the outgoing particle momenta is isotropic in azimuthal direction, i.e., for each value of the polar angle $\theta$ the value of the azimuthal angle $\varphi$ is distributed uniformly in the interval $0 \div 2 \pi$. See figure $1 b$. 
$>$ From the above kinematics, it follows that to select elastic events, you have to register two tracks of secondary particles and be sure that these tracks are oppositely directed. This condition specifies requirements to find the proper detectors for registration and selection of elastic processes in studies with colliding polarized $p p$ and $d d$ beams of the NICA collider.

\section{Spin Physics Detector and Monte Carlo simulations of scattering events}

The concept of the detector for spin physics studies at the NICA collider has been presented in [5]. The required set of devices for the Spin Physics Detector described in this publication can be also successfully used to register the elastic processes. The scheme of the detectors arrangement to register and select the elastic $p p, n p$ and $d d$ events is shown in figure 2a. Two-coordinate detectors - internal $(I T)$ and external trackers, determine the tracks of secondary particles, i.e., the directions of the scattered reaction products. Hodoscopes of scintillating counters $(H S C)$ and resistive plate chambers $(R P C)$ are proposed as trigger detectors for the data acquisition system $(D A Q)$.

The use of a solenoid magnet in the $S P D$ detector provides another powerful criterion for the selection of elastic events. The magnetic field of this magnet shifts the azimuthal $\varphi$ coordinates of secondary particles that allows one to carry out off-line data processing to determine and compare their momenta. The solenoid magnetic field does not affect $\theta$ polar coordinates of secondary particles and so there are no problems in setting the $\theta$ trigger.

To register and select of the elastic neutron-proton $(n p)$ events we can use a Range System ( $R S$ ) (particle range detectors) provided by the concept of $S P D$. The use of these detectors will allow us to estimate the energy value of the scattered neutrons and determine its approximate $\theta$ and $\varphi$ coordinates. The signal from the selected part of the range detectors will allow one to select and register the elastic $n p$ events included in the trigger condition.

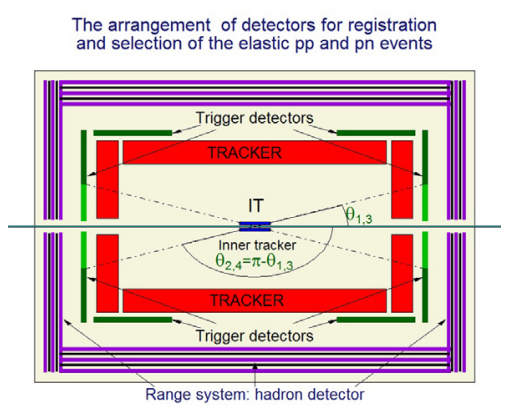

(a)

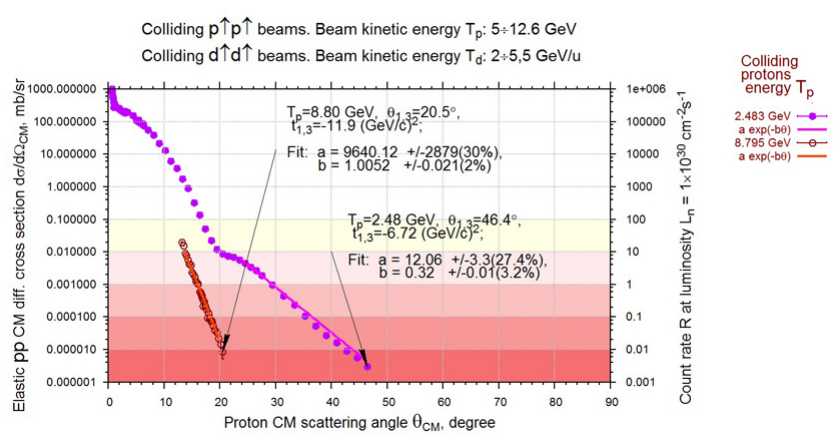

(b)

Figure 2. (a) The arrangement of detectors in the $S P D$ module for registration and selection of the elastic $p p, n p$ and $d d$ events. (b) Fit of the experimental data sets on the elastic $d \sigma / d \Omega(p p)$ at the $p$ beam energies of $T_{p}=2.48$ and $8.8 \mathrm{GeV}$.

Angular $\theta, \varphi$ dependencies of the elastic events detected by $S$ PD can be visualized by Monte Carlo modeling and used while preparing and carrying out these studies. The values of the parameters required for Monte Carlo simulations can be taken from available sets of experimental data $d \sigma / d \Omega(N N)$ in the Center of Mass (see the compilation [6]). Examples of the experimental $d \sigma / d \Omega(p p)$ and $d \sigma / d \Omega(n p)$ data from [6] are shown in figures $2 \mathrm{~b}$ and $5 \mathrm{c}$.

The scattering events registered by the $S P D$ coordinate detectors can be represented by a set of points - the coordinates of particles 3 and 4 in the $\theta, \varphi$ plane. Distributions of elastically scattered particles 3 and 4 in the $\theta$ coordinate can be approximated by exponential dependencies taken from the data [6] shown in figures $2 \mathrm{~b}$ and $5 \mathrm{c}$. The angular $\varphi$ distribution of the elastic events can be uniform. 
For inelastic scattered particles the $\theta$ and $\varphi$ angular distributions can also be uniform. Under the above assumptions generated by the procedure, the Monte Carlo angular $\theta, \varphi$ distributions of elastic and inelastic events without any selection criteria are shown in figure 3a. Here the black dots represent the inelastic events, red - elastic scattering of the proton from the red oncoming beam, and the blue dots - elastic scattering of the proton from the blue beam.

The counting rate $R$ of scattering events in the collider is proportional to the interaction cross section $\sigma_{\text {int }}$ and luminosity of the collider $L: R=\sigma_{\text {int }} \cdot L$. The total $p p$ cross sections in the energy range of the NICA collider are following: $\sigma_{\text {tot }} \approx 40 \mathrm{mb}, \sigma_{\text {tot,el }} \approx 10 \mathrm{mb}$ and $\sigma_{\text {tot, in }} \approx 30 \mathrm{mb}$. The estimated luminosity of the colliding beams of polarized protons will reach $L_{p p} \approx 1 \cdot 10^{32} \mathrm{~cm}^{-2} \mathrm{~s}^{-1}$ at beam energies more than $T_{p} \approx 11 \mathrm{GeV}$ [7]. At $T_{p} \approx 3 \mathrm{GeV}$ the luminosity is expected to be equal to $L_{p p} \approx 1 \cdot 10^{30} \mathrm{~cm}^{-2} \mathrm{~s}^{-1}$. In this paper, we consider the examples of nucleon-nucleon collisions at energies of colliding nucleons $T_{p}=2.48 \mathrm{CeV}, T_{p}=8.8 \mathrm{GeV}$ and $T_{n} \approx 1.43 \mathrm{GeV}$. Due to these estimates at $T_{p}=8.8 \mathrm{GeV}$ we have used the value $L_{p p} \approx 1 \cdot 10^{32} \mathrm{~cm}^{-2} \mathrm{~s}^{-1}$, and for the other energies the values of $L_{p p} \approx 1 \cdot 10^{30} \mathrm{~cm}^{-2} \mathrm{~s}^{-1}$. Then the expected counting rates will be $R_{e l} \approx 1 \cdot 10^{4} \mathrm{~s}^{-1}$ and

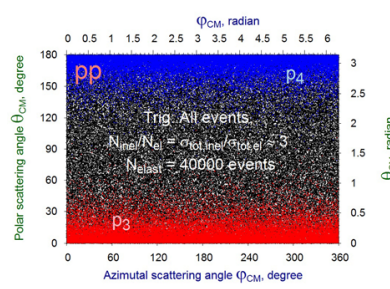

(a)

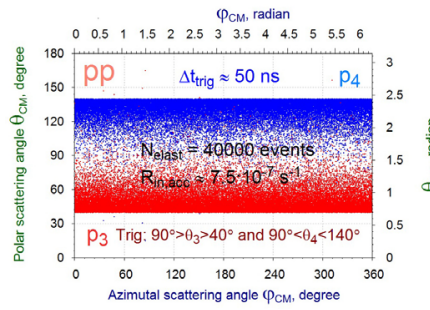

(b)

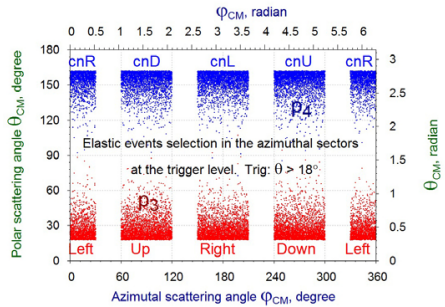

(c)

Figure 3. (a) Example of a Monte Carlo simulation for all the events without selection. (b) Simulation of elastic $p p$ events with two trigger conditions. (c) The left-right and up-down events selection in the azimuthal sectors at the trigger level.

$R_{\text {in }} \approx 3 \cdot 10^{4} \mathrm{~s}^{-1}$. When the trigger signal duration is $\Delta t \sim 50 \mathrm{~ns}$, the probability of the inelastic event presence in this interval is $p_{i n} \sim R_{i n} \cdot 5 \cdot 10^{-8}=1.5 \cdot 10^{-3}$ and of the elastic ones is $p_{e l} \sim 5 \cdot 10^{-4}$. The counting rate of accidental coincidences, i.e. the probability of detecting the inelastic events along with the elastic ones with trigger conditions for the given scattering angle $C 3$ : $90^{\circ} \geq \theta_{3} \geq C_{3}^{\circ}$ and $90^{\circ} \leq \theta_{4} \leq \pi-C_{3}^{\circ}$, will be $R_{a c c} \approx p_{e l} \cdot R_{i n} \approx 7.5 \cdot 10^{-7} s^{-} 1$. This level of accidentals leads to almost complete absence of inelastic events in the following figures for the results of the $M C$ simulations.

Figures $3 \mathrm{~b}$ and $3 \mathrm{c}$ show examples of using different types of trigger conditions for the elastic $p p$ events selected in the $\theta, \varphi$ plane. Selection of the elastic events in the given range only of the polar angles $\theta_{3,4}$ (fig. $3 \mathrm{~b}$ ) provides the maximum event rate. In $\vec{d} \vec{d}$ collisions to measure all the components of polarization observables, it is required to measure the left-right and up-down asymmetries of the outputs of the elastic process. In this case, the trigger detectors must identify separately $\Delta \varphi$ zones of scattering particles of the "red" beam to the left, right, up and down in the forward hemisphere $\left(0<\theta<90^{\circ}\right)$ and the corresponding $\Delta \varphi$ areas of conjugated particles of the "blue" beam scattered in the back hemisphere $\left(90^{\circ}<\theta<180^{\circ}\right)$.

\section{Examples of Monte Carlo simulation for $p p$ and $n p$ collisions}

By fitting the experimental $\theta$ angular dependencies of $d \sigma / d \Omega(p p)$ and $d \sigma / d \Omega(n p)$ [6] from figure $2 \mathrm{~b}$ and $5 \mathrm{c}$, we get the values of the slope parameters of exponents to use Monte Carlo visualization for these processes. The values of $d \sigma / d \Omega$ from these data at the maximal angle $\theta_{\max }$ allow us to estimate the event counting rate at this angle. The results of Monte Carlo simulations for the energies of colliding protons of $T_{p}=2.48 \mathrm{GeV}$ and $T_{p}=8.8 \mathrm{GeV}$ are given in figure 4 and figure 5a. Figure $4 \mathrm{a}$ shows the dependence of $t_{1,3}(\theta)$ in the range of $0 \leq \theta \leq 90^{\circ}$ for $d \sigma / d \Omega(p p)$ data at 


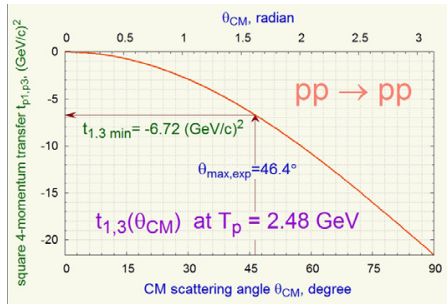

(a)

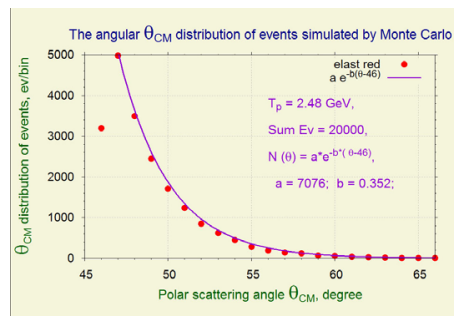

(b)

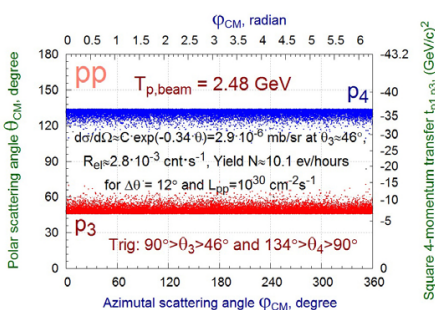

(c)

Figure 4. (a) The range of $t_{1,3}\left(\theta_{3}\right)$ values when $0 \leq \theta_{3} \leq 90^{\circ}$ for $p p$ at $T_{p}=2.48 \mathrm{GeV}$. (b) The $\theta_{C M}$ distribution of the events simulated by MC procedure at $T_{p}=2.48 \mathrm{GeV}$. (c) Results of the MC simulations for $p p$ at $T_{p}=2.48 \mathrm{GeV}$.

$T_{p}=2.48 \mathrm{GeV}$. The maximal angle and the corresponding $t_{1,3}\left(\theta_{\max }\right)$ value are indicated. Generated by the $M C$ procedure the $\theta$ distributions of elastic and quasi-elastic events are shown in figure $4 \mathrm{~b}$ for $p p$ collisions at $T_{p}=2.48 \mathrm{GeV}$, and in figure $6 \mathrm{a}$ for $n p$ collisions at $T_{n}=1.427 \mathrm{GeV}$. Figs. $4 \mathrm{c}, 5 \mathrm{a}, 6 \mathrm{~b}$, and $6 \mathrm{c}$ show the $\mathrm{MC}$ generated $\theta, \varphi$ distributions of the elastic events and the expected counting rates $R(\theta)=L \iint d \sigma / d \Omega(\theta) \cdot \sin \theta \cdot d \theta d \varphi \simeq 2 \pi L d \sigma / d \Omega(\theta) \cdot \sin \theta \cdot \Delta \theta\left[s^{-1}\right]$ and yields $Y(\theta)=R(\theta) \cdot 3.6 \cdot 10^{3}\left[h^{-1}\right]$ in the specified band $\left[\theta_{\max } \div \theta_{\max }+\Delta \theta\right]$ at the number of $T_{\text {beam }}$ values.

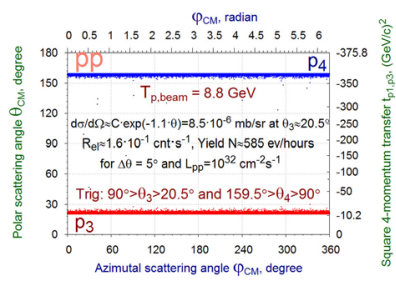

(a)

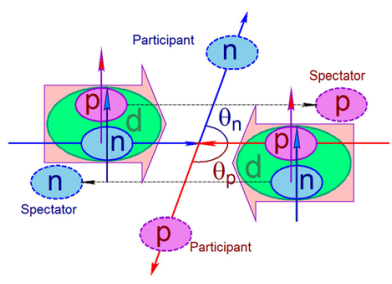

(b)

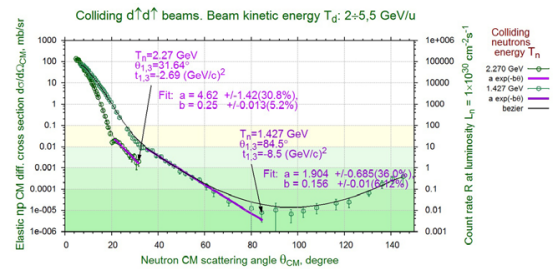

(c)

Figure 5. (a) Results of the Monte Carlo simulations for $p p$ collisions at $T_{p}=8.8 \mathrm{GeV}$. (b) The quasi-elastic $\vec{N} \vec{N}$ scattering in the polarized deuteron $\vec{d} \vec{d}$ collisions. (c) Fit of the experimental data sets on $d \sigma / d \Omega$ for elastic $n p$ scattering at the energies of the colliding $d d$ beams at $T_{d}=2.85$ and $4.54 \mathrm{GeV}$.

Assuming that the deuteron is a weakly bounded system, one can apply the formalism of elastic $p p$ scattering to describe a quasi-elastic $N N$ scattering independently on each nucleon in the deuteron. See figure $5 \mathrm{~b}$. The nucleons in the deuteron have the same value and orientation of polarization as the

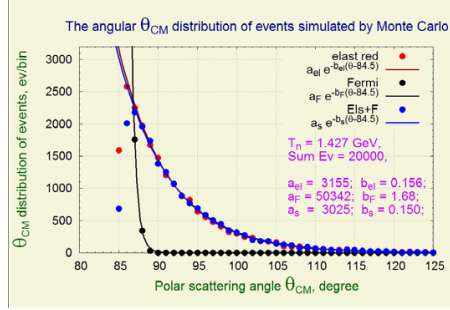

(a)

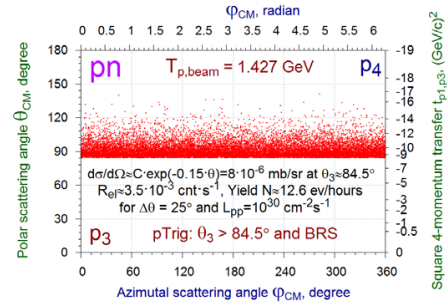

(b)

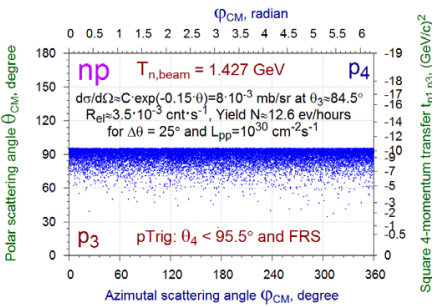

(c)

Figure 6. (a) The $\theta_{C M}$ distribution of events simulated by the MC procedure at $T_{n}=1.427 \mathrm{GeV}$. (b) Results of the MC simulations for $p n$ collisions at $T_{n}=1.427 \mathrm{GeV}$. (c) The same but for $n p$ collisions.

deuteron itself $\vec{P}_{N}=\vec{P}_{d}$ and half deuteron kinetic energy $T_{N}=0.5 T_{d}$. The Fermi motion of nucleons in the deuteron influences weakly on the angular distribution of scattering products $\Delta \theta_{F m}<22 \mathrm{mrad}$ at $p_{p} \sim 2.2 \mathrm{GeV} / \mathrm{c}$. The Gaussian momentum spread of the nucleon in the deuteron is also small $\sigma_{p} \approx 2.5 \%$. Results of the MC simulations for $p n$ and $n p$ collisions at $T_{n}=1.427 \mathrm{GeV}$ are presented in figure 6. Figure 6a shows the $\theta$ distributions simulated by the $M C$ procedure at $T_{n}=1.427 \mathrm{GeV}$ 
for: - elastic $n p$ scattering (red dots and curve); - due to the Fermi motion of the nucleons in the deuteron (black dots and curve); and - elastic scattering + Fermi motion (blue dots and curve). The effect of Fermi motion is noticeable only within the limits of its $\theta$ distribution. Trigger conditions for the distributions of figure $6 \mathrm{~b}$ and $6 \mathrm{c}$ include $H S C$ or/and $R P C$ signal $\theta_{3}>84.5^{\circ}$ from the $p n$ scattered proton and the signal of the scattered neutron from the corresponding backward part of the $R S$ detectors - BRS and vice versa for the $n p$ scattering.

\section{Conclusion}

The features of the kinematics of the processes of elastic $p p$ and $d d$ scattering in the collider system have been considered. The equality and opposite direction of the momenta of the scattered particles provide a powerful selection criterion for elastic collisions in this system. Possible choices of the trigger signal to register the tracks of secondary particles and data acquisition system have been given.

According to available $d \sigma / d \Omega_{C M}(p p)$ and $d \sigma / d \Omega_{C M}(n p)$ data in the energy range of the NICA collider we have estimated the parameters of the angular distributions for the Monte Carlo simulations of these processes. The results of $M C$ simulations of the elastic and quasi-elastic $p p$ and $n p$ processes and estimations of counting rates and outputs of these reactions at specified scattering angles have been given. The presented examples of the $M C$ simulations of elastic events can be used in the research of elastic $p p, n p$, and $d d$ interactions in the colliding beams at the NICA collider. The obtained results have shown that in the colliding $p p$ and $d d$ beams at the NICA collider can obtain a sufficiently high counting rate and outputs of the elastic collision events.

Acknowledgements

The author is grateful to his colleague E.V. Chernykh for his help to prepare the talk and this contribution.

\section{References}

[1] NICA - Nuclotron - based Ion Collider fAcility - a Base for Fundamental Research and Innovation Development, (JINR, Dubna 2014). http://nica.jinr.ru/files/Booklet-NICA.pdf

[2] V.I. Sharov, Proceedings of "XV Advanced Research Workshop on High Energy Spin Physics. (DSPIN-13)", Dubna, October 8-12, 2013, edited by A.V. Efremov, S.V. Goloskokov, JINR, E1,2-2014-12, (JINR, Dubna, 2014), 367-370

[3] V.I. Sharov, Proceedings of "XXII International Baldin Seminar on High Energy Physics Problems. Baldin ISHEPP XXII", Dubna, September 15-20, 2014, PoS (Baldin ISHEPP XXII) 102. http://pos.sissa

[4] V.I. Sharov, Proceedings of "XVI Workshop on High Energy Spin Physics (D-SPIN2015)". Dubna, September 15-20, 2014, Journal of Physics: Conference Series 678, 012011 (2016)

[5] I.A. Savin, A.V. Efremov et al., Spin Physics Experiments at NICA-SPD with polarized proton and deuteron beams, Letter of Intent. (2014)

[6] J. Bystricky and F. Lehar, Nucleon-Nucleon Scattering Data, editors H. Behrens and G. Ebel (Fachinformationszentrum Karlsruhe, 1981 Edition, Nr. 11-2 and Nr. 11-3, 1981)

[7] A.D. Kovalenko, Proceedings of "XV Advanced Research Workshop on High Energy Spin Physics. (DSPIN-13)", Dubna, October 8-12, 2013, edited by A.V. Efremov, S.V. Goloskokov, JINR, E1,2-2014-12 (JINR, Dubna, 2014) 345-350 